\title{
JC Virus Replication at the First Symptoms of Multiple Sclerosis: A Case Report
}

\author{
Serena Delbue ${ }^{a}$ Carlo Sebastiano Tadeo $^{b}$ Francesca Elia $^{a}$ \\ Pasquale Ferrante ${ }^{a, c}$ \\ ${ }^{a}$ Department of Biomedical, Surgical and Dental Sciences, University of Milan, and ${ }^{b}$ Neurology Department, \\ Istituto Clinico Città Studi, Milan, and ' Fondazione Ettore Sansavini, Health Science Foundation, Lugo, Italy
}

\section{Key Words}

Multiple sclerosis · JC virus · Triggering agent

\begin{abstract}
Multiple sclerosis (MS) is an autoimmune, demyelinating disorder of unknown etiology, in which viruses have been suggested as etiological/triggering agents. The attention to the association between viruses and MS has been rekindled by the development of progressive multifocal leukoencephalopathy in natalizumab-treated MS patients. Here we report the case of a woman with JC virus (JCV) replication in the cerebrospinal fluid, blood and urine collected at the first symptoms of MS and during several follow-up visits. This observation shows that JCV can be associated with MS without a relation with natalizumab treatment, although the triggering role of JCV in some cases of MS will require further studies.

(c) 2015 S. Karger AG, Basel
\end{abstract}

\section{Introduction}

Multiple Sclerosis (MS) is a chronic neurological disorder characterized by a variable clinical course and pathologically by the progressive accumulation of plaques of demyelination within the white matter of the central nervous system (CNS). The etiology of this autoimmune disease is still unknown, but genetic and environmental factors are thought to play a significant role in its pathogenesis [1]. Among the environmental factors, evidence that viruses may cause or participate in the pathogenesis of MS comes from many epidemiological studies that suggested that possible exogenous factors may be associated with MS $[2,3]$. The possibility that MS could be triggered by infectious agents was already suggested by Pierre Marie, a Charcot assistant, at the time of its clinical definition in the 19th century, but despite all the scientific efforts, the virus or viruses responsible for the disease have not yet been identified. The human polyomavirus, JC virus (JCV), is the recognized etiological agent of another demyelinating disease of the CNS, the progressive multifocal leukoencephalopathy (PML). Some similarities between MS and PML and also the specific features of JCV, which is neurotropic, can be persistent in the host and subjected to asymptomatic periodical reactivation have prompted an investigation into the association of MS with JCV reactivation [4]. Additionally, the association of MS natalizumab treatment with the development of more than 500 cases of PML worldwide has reawakened the scientific interest in the relationship between JCV and MS [5]. Here we report the case of a patient with

\section{KARGER 125}

(c) 2015 S. Karger AG, Base

$0300-5526 / 15 / 0585-0278 \$ 39.50 / 0$

E-Mail karger@karger.com

www.karger.com/int
Pasquale Ferrante, $\mathrm{MD}, \mathrm{PhD}$

Department of Biomedical Surgical and Dental Sciences

University of Milan, Via Pascal, 36

IT-20133 Milan (Italy)

E-Mail pasquale.ferrante@unimi.it 
Table 1. JCV genome presence, viral load and antibodies in clinical specimens collected from the patient at different times after the first MS symptom onset

\begin{tabular}{|c|c|c|c|c|c|}
\hline & $\begin{array}{l}\text { T1 } \\
\text { November 8, } 2013\end{array}$ & $\begin{array}{l}\text { T2 } \\
\text { November 21, } 2013\end{array}$ & $\begin{array}{l}\text { T3 } \\
\text { February 4, } 2014\end{array}$ & $\begin{array}{l}\text { T4 } \\
\text { August 6, } 2014\end{array}$ & $\begin{array}{l}\text { T5 } \\
\text { September 30, } 2014\end{array}$ \\
\hline \multicolumn{6}{|c|}{ JCV DNA presence and viral load } \\
\hline CSF & 210 copies $/ \mathrm{ml}$ & not done & not done & negative & not done \\
\hline PBMCs & 12 copies $/ \mathrm{ml}$ & 10 copies $/ \mathrm{ml}$ & negative & negative & negative \\
\hline Urine & 4,900 copies $/ \mathrm{ml}$ & 1,500 copies $/ \mathrm{ml}$ & 720,000 copies $/ \mathrm{ml}$ & 6,900 copies $/ \mathrm{ml}$ & 138,000 copies $/ \mathrm{ml}$ \\
\hline Serum & 160 copies $/ \mathrm{ml}$ & negative & negative & 130 copies $/ \mathrm{ml}$ & negative \\
\hline \multicolumn{6}{|c|}{ Anti JCV-IgG (OD; negative control: 0.1) } \\
\hline CSF & 0.27 & not done & not done & 0.19 & not done \\
\hline Serum & 0.19 & 0.25 & 0.26 & 0.22 & 0.26 \\
\hline
\end{tabular}

typical clinical and neurological MS associated with JCV viremia, viruria and active replication in the CNS at the moment of clinical onset. This almost unique observation shows that JCV can be associated with MS without a relation with natalizumab treatment, renewing the hypothesis of an implication of JCV in triggering MS.

\section{Case Description}

A patient, complaining of a 1-month history of dysesthesia, motor weakness of the four limbs and fatigability when walking, was evaluated at the Istituto Clinico Città Studi, Milan, Italy in November 2013. Neurological examinations with the manual muscle test revealed no motor weakness and no sensory deficits. The patient presented hyperreflexia of the lower limbs and multifocal white matter disease areas of increased T2 signal in both cerebral hemispheres, around the corpus callosum, without contrast. Spinal cord MRI revealed a lesion within the cervical cord C2 level, with contrast (fig. 1a). Oligoclonal bands were observed in the cerebrospinal fluid (CSF) but not in the serum; blood cells in the CSF were $6 / \mathrm{mm}^{3}$. HIV antibodies and vasculitis screening tests were negative, and immunoglobulin count and lymphocytic typing were within the normal range. A diagnosis of clinically isolated syndrome was made, according to McDonald's criteria [6]. The patient has had continuous follow-up and was asymptomatic, with no new lesions at MRI, until February 2014, when she was suffering from lumbar dysesthesia, without motor weakness. At that time new lesions in the cervical spine and conus medullaris were detected, and enhanced lesions with contrast in the thalamus, besides the other spinal lesions, did not take up gadolinium (fig. 1b). In September 2014, additional new lesions were present at MRI (fig. 1c). The diagnosis of MS was definitively made [7]. PML diagnosis was both radiologically and clinically excluded for the following reasons: (1) MS lesions usually occur in both gray and white matter and in juxtacortical locations, and the vast majority do not enhance at a given time [4]; (2) PML lesions exhibit faint contrast enhancement and have less well-defined margins that extend along gray-white matter junctions within the cortex; (3) PML is characterized by a progres-

JC Virus and Multiple Sclerosis sive monophasic clinical course, while the patient suffers from a relapse/remitting disease, and (4) oligoclonal bands observed in the CSF are typical of MS. In November 2013, the presence of neurotropic viruses in the CSF, including herpes simplex viruses $1 / 2$, varicella zoster virus, Epstein-Barr virus, human herpesviruses $6 \mathrm{~A} / \mathrm{B}$ and human polyomaviruses JCV and BKV, was evaluated by specific quantitative real-time polymerase chain reaction assays. JCV DNA was found and quantified as 210 copies $/ \mathrm{ml}$, while no evidence of the genomes of the other viruses was present. Additionally, JCV DNA was found in peripheral blood mononuclear cells (PBMCs, 12 copies $/ \mathrm{ml})$, serum $(160$ copies $/ \mathrm{ml})$ and urine samples $(4,900$ copies $/ \mathrm{ml}$ ). Subsequently, the clinical specimens were periodically analyzed (November 21, 2013; February 4, 2014; August 6, 2014; September 30,2014) after obtaining the patient's informed consent; urine samples were always positive for the presence of the JCV genome, while PBMCs and serum were alternatively positive and negative (table 1). JCV strain was defined as an archetype IS strain, with some deletions and point mutations, and as genotype $1 \mathrm{~A}$, in both the CSF and urine samples (fig. 2). Moreover, antibodies against JCV were detected in the collected CSF and sera (table 1).

\section{Discussion}

The issues regarding the implications of infectious agents for the etiology of MS have not found any definitive answers, although theories and hypotheses have been formulating since more than 150 years ago. The most substantiated definition of MS states that it is an autoimmune phenomenon occurring in genetically predisposed individuals, but this does not rule out the possible action, as etiological or triggering factors, of one or more infectious agents, including a virus. In 1884, Pierre Marie was certain that the cause of MS was a still unknown infective agent, fightable with a vaccine [8]. Since that time a long list of candidate viruses that could be potential triggers of the onset or the recurrence of MS has been produced - 

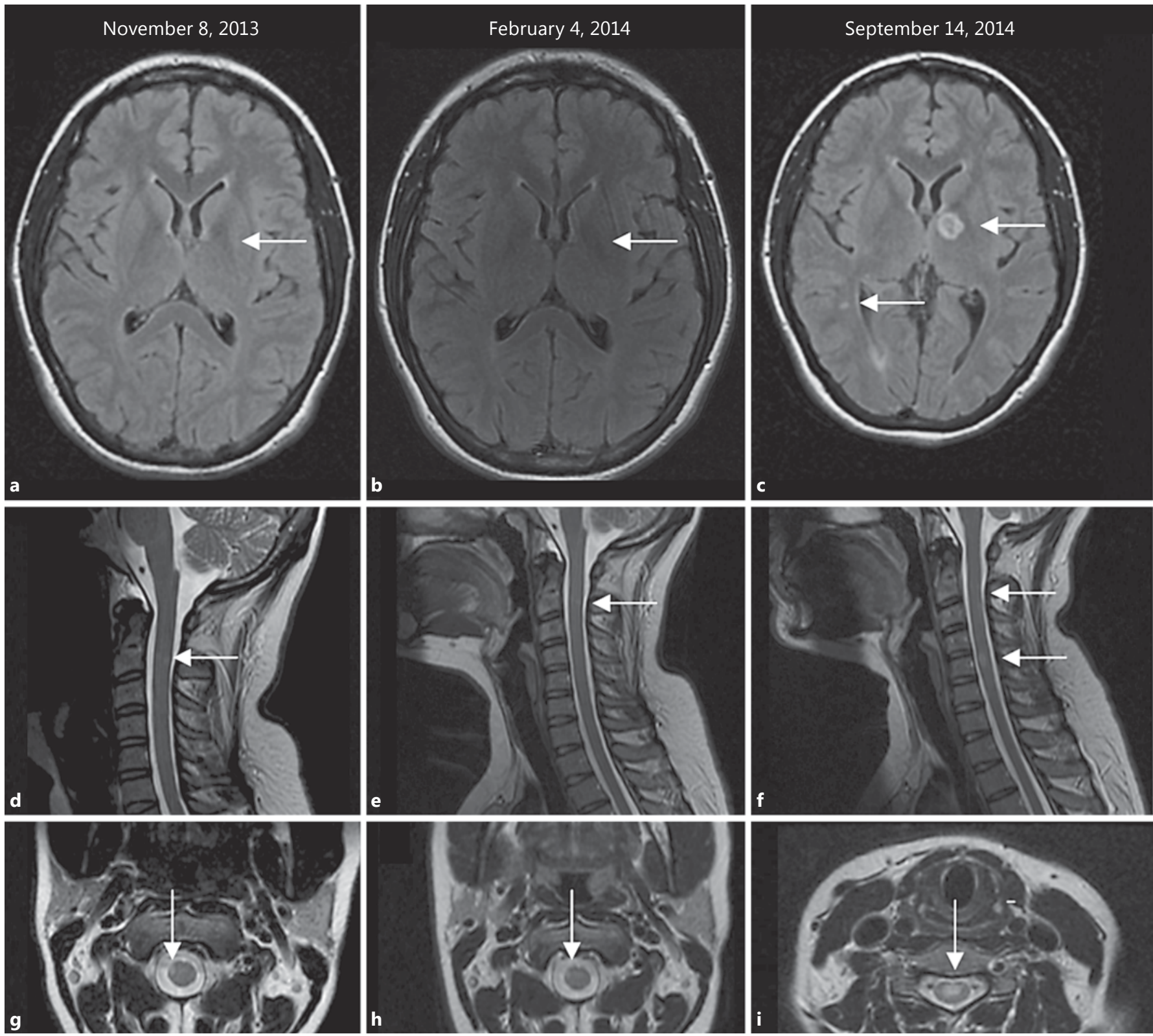

Fig. 1. Brain and spinal cord MRI images taken at the initial visit and during two follow up visits. MRI images were taken in November 2013 (a, d, g; onset): the arrows indicate a little lesion in the corpus callosum and in the cervical spinal cord, February 2014 (b, e, h; follow-up): the arrows indicate the same lesion, and September 2014 (c, $\mathbf{f}, \mathbf{i}$; relapses): the arrows indicate new lesions in the corona radiate, internal capsule and cervical spinal cord.

human herpesviruses, Theiler virus, paramyxovirus, coronavirus, cardiovirus, enteroviruses and human retroviruses - but so far none of them has been identified as directly implicated in the pathogenesis of MS [3].

In 1991, Gerard Stoner was the first to make some important observations regarding the human polyomavirus JCV, which was known to be the etiological agent of the demyelinating disease, PML. In particular, he proposed that 'the pathogenetic immune response in a MS brain may be directed predominantly toward antigens of JCV which is latent in glial cells'. JCV is difficult to be cultivated in vitro and this fits the 'noncultivable' criterion for an MS agent [9]. It can replicate in the oligodendrocytes, causing cells lysis and subsequent demyelination. It is 
Fig. 2. Schematic representation of the JCV, NCCR fragment, nucleotide sequence. The JCV detected in both the CSF and the urine samples was identified as an IS strain (CY, GI:409080), with the deletions of 14 and 3 bases in the CSF and urine isolates, respectively, and with some point mutations.

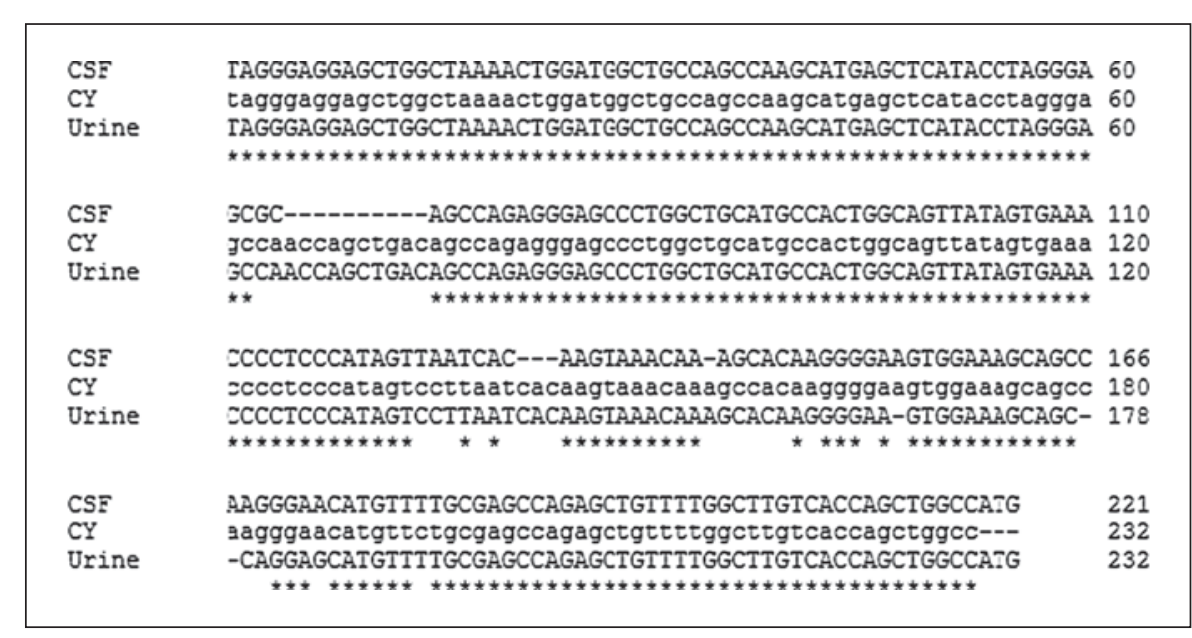

ubiquitous and very frequent in the worldwide population, mainly associated with asymptomatic infection and reactivation, and is able to establish latency in kidney and lymphocytes - potential reservoirs of the virus in the host $[10,11]$. All these above-mentioned characteristics, which are intrinsic in its own biology, make JCV one of the best candidates for being associated with MS.

However, there are two principal questions that need to be addressed in order to define the potential role of JCV in the onset of MS: (1) does the JCV in the CSF play a relevant role in the development of the MS demyelinating lesions? and (2) is the JCV already latent in the brain or is it carried from the kidneys?

The intriguing scientific debate regarding the role of early JCV infection in the development of MS is wide and still ongoing and the answer to the first question is far from being found. Some studies did not detect virus DNA in MS patients [12], but several others successfully detected the viral genome in CNS samples from MS patients [13]. Alvarez-Lafuente et al. [14] collected CSF samples from MS patients at the first demyelinating event, finding JCV DNA in two CSF samples.

Regarding the site of JCV latency, it is commonly believed that JCV causes PML after reactivating in an immunocompromised setting, from the kidney, its main host site of latency, to the CNS. However, there is also much evidence that may support the hypothesis of an asymptomatic JCV persistent infection of the CNS, reporting the presence of the viral genome and the selective expression of some proteins in the CSF and in the brain tissue of patients affected with non-PML neurological diseases and also of healthy subjects [15-18].

JC Virus and Multiple Sclerosis
From the natalizumab lesson, we learned that the block of cell traffic to the brain may be associated with the development of PML, due to the infection/reactivation of JCV. In this case, the decrease of immune surveillance in the CNS may be the cause of the reactivation of the virus, which is already latent in the brain [19].

Consequently, as Stoner [9] proposed, the host's immune response to antigens produced during latency or following abortive reactivation may trigger an immunemediated process of demyelination at the onset of MS.

The initial case of MS herein described adds further elements in support of the idea that JCV can be considered as one of the best candidates for being associated with the pathogenesis of MS. It is interesting to underline that JCV DNA was detected in the CSF of the patient in concomitance with the first clinical and radiological appearance of the disease, but 9 months later the viral genome was not found in the CSF, thus suggesting a possible hit-and-run mechanism of action of JCV. It is likely that the JCV DNA load decreased during the time in the blood samples, both in PBMCs and serum, but it was always detected at high levels in the urine samples. The presence of overall increasing titers in serum anti-JCV IgG and of decreasing titers in the CSF may suggest that the patient was experiencing a primary JCV infection. Unfortunately, the lack of an assay for testing JCV IgM antibodies prevented the establishment of this. However, taken together, the observations on antibody titers and viral loads lead us to speculate that the virus was replicating in all the body areas in concomitance with the onset of the disease, and the replication was then compartmentalized in the kidneys, where JCV remains throughout, alternating latency and active replication. 
Molecular characterization of the JCV strain found in the CSF showed the presence of the CY molecular organization. This observation, even if rare, is not unique since it has been previously reported in a subset of PML patients with a relatively good immunological status, as for the MS case we are reporting here [20].

In summary, even if we cannot rule out the presence of the viral genome in the CSF and in all the other body fluids examined as a simple bystander, the present observations indicate that JCV may be an important factor in triggering MS and/or in its etiology, at least in some clinical cases, and thus further attempts should be made to clarify the role of this intriguing agent in MS.

\section{Acknowledgments}

We thank the patient for consenting to the publication of her medical history. This work was partially supported by a grant from the Italian Ministry of Education, University and Research (PRIN 2010-2011) to Pasquale Ferrante.

\section{Disclosure Statement}

The authors declare no conflicts of interest.

\section{References}

1 Compston A, Coles A: Multiple sclerosis. Lancet 2008;372:1502-1517.

2 Granieri E, Casetta I, Tola MR, et al: Multiple sclerosis: infectious hypothesis. Neurol Sci 2001;22:179-185.

3 Ascherio A, Munger KL: Environmental risk factors for multiple sclerosis. Part I. The role of infection. Ann Neurol 2007;61:288-224.

4 Khalili K, White MK, Lublin F, Ferrante P, et al: Reactivation of JC virus and development of PML in patients with multiple sclerosis. Neurology 2007;68:985- 990.

5 TYSABRI Safety Update (2015). Available at: https://medinfo.biogenidec.com/medinfo/ secure/pmlresource. do? resource $=$ TYSABRI PMLSafetyUpdate (accessed May 1, 2015).

6 Polman CH, Reingold SC, Banwell B, et al. Diagnostic criteria for multiple sclerosis: 2010 revisions to the McDonald criteria. Ann Neurol 2011;69:292-302.

7 Miller DH, Barkhof F, Montalban X, et al. Clinically isolated syndromes suggestive of MS. Part 1. Natural history, pathogenesis, diagnosis and treatment. Lancet Neurol 2005;4: 281-288.

8 Pierre Marie: Sclérose en plaques et maladie infecteuses. Prog Med 1884;12:287-289.
9 Stoner GL: Implications of progressive multifocal leukoencephalopathy and JC virus for the etiology of MS. Acta Neurol Scand 1991; 83:20-33.

10 Ferenczy MW, Marshall LJ, Nelson CD, et al: Molecular biology, epidemiology, and pathogenesis of progressive multifocal leukoencephalopathy, the JC virus-induced demyelinating disease of the human brain. Clin Microbiol Rev 2012;25:471-506.

11 Hirsch HH, Kardas P, Kranz D, Leboeuf C: The human JC polyomavirus (JCPyV): virological background and clinical implications. APMIS 2013;121:685-727.

12 Yousry TA, Major EO, Ryschkewitsch C, et al: Evaluation of patients treated with natalizumab for progressive multifocal leukoencephalopathy. N Engl J Med 2006;354:924-933.

13 Ferrante P, Omodeo-Zorini E, Caldarelli-Stefano R, et al: Detection of JC virus DNA in cerebrospinal fluid from multiple sclerosis patients. Mult Scler 1998;4:49-54.

14 Alvarez-Lafuente R, García-Montojo M, De Las Heras V, et al: JC virus in cerebrospinal fluid samples of multiple sclerosis patients at the first demyelinating event. Mult Scler 2007; 13:590-595.
15 White FA 3rd, Ishaq M, Stoner GL, Frisque RJ: JC virus DNA is present in many human brain samples from patients without progressive multifocal leukoencephalopathy. J Virol 1992;66:5726-5734.

16 Delbue S, Branchetti E, Boldorini R, et al: Presence and expression of JCV early gene large $\mathrm{T}$ antigen in the brains of immunocompromised and immunocompetent individuals. J Med Virol 2008;80:2147-2152.

17 Perez-Liz G, Del Valle L, Gentilella A, et al: Detection of JC virus DNA fragments but not proteins in normal brain tissue. Ann Neurol 2008;64:379-387.

18 Tan CS, Ellis LC, Wüthrich C, et al: JC virus latency in the brain and extraneural organs of patients with and without progressive multifocal leukoencephalopathy. J Virol 2010;84: 9200-9209.

19 Ransohoff RM, Kivisäkk P, Kidd G: Three or more routes for leukocyte migration into the central nervous system. Nat Rev Immunol 2003;3:569-581.

20 Ferrante P, Delbue S, Pagani E, et al: Analysis of JC virus genotype distribution and transcriptional control region rearrangements in human immunodeficiency virus-positive progressive multifocal leukoencephalopathy patients with and without highly active antiretroviral treatment. J Neurovirol 2003;9:4246. 